\title{
"Escape" of Aldosterone Production in Patients with Left Ventricular Dysfunction Treated with an Angiotensin Converting Enzyme Inhibitor: Implications for Therapy
}

\author{
Bertram Pitt \\ Division of Cardiology, Department of Internal Medicine, \\ University of Michigan Medical Center, Ann Arbor, Michigan, USA
}

Summary. Despite the findings in randomized trials of a significant effect of angiotensin-converting enzyme (ACE) inhibitors in reducing morbidity and mortality of patients with symptomatic left ventricular dysfunction, the morbidity and mortality of these patients remains relatively high. One potential strategy to further improve morbidity and mortality in these patients is blockade of aldosterone. Many clinicians have assumed that ACE inhibitors would block both angiotensin II and aldosterone. However, there are data to suggest that aldosterone production may "escape" despite the use of an ACE inhibitor. An escape of aldosterone production has several important consequences, including: sodium retention, potassium and magnesium loss, myocardial collagen production, ventricular hypertrophy, myocardial norepinephrine release, endothelial dysfunction, and a decrease in serum high density lipoprotein cholesterol. Due to the potential importance of these mechanisms, the finding that there is a significant correlation between aldosterone production and mortality in patients with heart failure, as well as evidence that an aldosterone antagonist, spironolactone, when administered to patients with heart failure treated with conventional therapy including an ACE inhibitor results in increased diuresis and symptomatic improvement, an international prospective multicenter study has been organized, the Randomized Aldactone Evaluation Study (RALES Pilot Study), to evaluate the safety of blocking the effects of aldosterone in patients with heart failure treated with an ACE inhibitor.

Cardiovasc Drugs Ther 1995;9:145-149

Key Words. heart failure, aldosterone, spironolactone, left ventricular dysfunction, angiotensin II, angiotensin converting enzyme inhibitors

Large-scale randomized trials such as the Cooperative North Scandinavian Enalapril Survival Study (Consensus) [1], Studies of Left Ventricular Dysfunction (SOLVD) Treatment Trial [2], Vasodilator-Heart Failure Trial (V-HeFT-II) [3], Survival After Left Ventricular Enlargement (SAVE) [4], and Acute Infarction Ramipril Efficacy (AIRE) Study [5], have confirmed the effectiveness of angiotensin-converting enzyme (ACE) inhibitors in reducing total mortality and morbidity in patients with symptomatic left ventricular dysfunction early and late postinfarction as well as in chronic heart failure. There is also evidence suggesting that ACE inhibitors may have an effect in reducing the incidence of recurrent myocardial infarction in patients with left ventricular dysfunction $[4,6]$. On the basis of these studies and previous studies showing that ACE inhibitors improve exercise performance and symptoms in patients with symptomatic left ventricular dysfunction [7,8], ACE inhibitors in conjunction with diuretics with or without concomitant therapy with digoxin have become the standard for care of patients with symptomatic left ventricular dysfunction unless contraindicated because of hyperkalemia or not tolerated because of symptomatic hypotension, progressive renal dysfunction, angioedema, or intractable cough. The finding in randomized trials of a significant effect of ACE inhibitors on mortality and morbidity in patients with symptomatic left ventricular dysfunction has prompted efforts to apply this strategy to the vast majority of patients with asymptomatic left ventricular dysfunction, who have up to now not been treated with an ACE inhibitor.

While efforts to extend the use of ACE inhibitors to patients with asymptomatic left ventricular dysfunction are important, there is also evidence suggesting that despite the use of ACE inhibitors in patients with symptomatic left ventricular dysfunction, there remains a relatively high incidence of mortality and recurrent heart failure [2]. This realization has prompted efforts to explore other mechanisms and treatment strategies, including the use of betaadrenergic receptor blocking agents, inotropic agents, direct-acting vasodilators, and metabolically effective

Address for correspondence: Bertram Pitt, M.D., Division of Cardiology, University Hospital, 1500 E. Medical Center Drive, Ann Arbor, MI 48109-0366, USA.

Received 14 March 1994, accepted in revised form 11 August 1994 
agents, to further reduce mortality and morbidity [9]. One potential strategy to further improve mortality and morbidity in the patient with symptomatic left ventricular dysfunction has been largely overlooked, that is, blockade of aldosterone.

Many clinicians have assumed that ACE inhibitor therapy would block both angiotensin II and aldosterone. However, there are data to suggest that aldosterone production remains important despite the use of an ACE inhibitor. After several months of ACE inhibitor therapy and continued inhibition of angiotensin II production in patients with hypertension, aldosterone levels may increase or "escape" [10]. A similar escape of aldosterone production has recently been shown in patients with heart failure treated with an ACE inhibitor [11]. Recent studies have also shown that relatively high doses of an ACE inhibitor, such as captopril $50 \mathrm{mg}$ three times daily for several days, suppresses resting levels of both angiotensin II and aldosterone, but during exercise both angiotensin II and aldosterone levels increase [12].

In another recent study using zofinopril in patients with acute myocardial infarction, it was shown that while ACE levels were almost completely suppressed angiotensin II and aldosterone levels escaped after several days [13]. The SOLVD trial has also suggested an escape of angiotensin II and catecholamine levels despite treatment with enalapril in doses of up to $10 \mathrm{mg}$ bid after 1 year [14]. Although aldosterone levels were not measured in this study, it can be postulated that if angiotensin II levels increase there would also be an increase in aldosterone levels. Thus there appears to be an "escape" of aldosterone production, and in some instances angiotensin II, despite continued use of an ACE inhibitor. The mechanisms for the "escape" of aldosterone and angiotensin II are incompletely understood. Alternative pathways for the production of angiotensin II have been identified $[15,16]$ and may become important after prolonged blockade of ACE, with a resultant increase in renin. Aldosterone production may be stimulated by decreased plasma levels of potassium and magnesium, and increased levels of ACTH, independent of the level of angiotensin II $[17,18]$.

Aldosterone is produced in the zona glomerulosa of the adrenal gland [17-20]. Plasma aldosterone levels are normally between 5 and $15 \mathrm{ng} / \mathrm{dl}$. Synthesis of aldosterone is mainly determined by angiotensin II. Other factors, such as an increase in ACTH, norepinephrine, or atrial naturietic factor, or a decrease in intracellular potassium and magnesium, are also of importance and may be critical in determining aldosterone synthesis in situations in which angiotensin II production is inhibited. Inactivation of aldosterone occurs mainly in the liver. Its metabolite tetrahydroaldosterone is conjugated with glucuronic acid and excreted in the urine. Impaired hepatic blood flow and metabolism of aldosterone in the liver may also be of importance in determining serum aldosterone levels.
In situations such as chronic heart failure, in which hepatic blood flow and metabolism are impaired, the plasma half-life of aldosterone may increase from a normal value of 30-35 minutes to over 70 minutes, with a concomitant increase in aldosterone levels from a normal range of $5-15 \mathrm{ng} / \mathrm{dl}$ to over $30 \mathrm{ng} / \mathrm{dl}$. Conversely, heparin, which blocks angiotensin II receptors in the zona glomerulosa of the adrenal gland and digitalis, through action on sodium/potassium ATPase, intracellular sodium, and potassium concentration, may decrease aldosterone production $[17,18]$.

The consequences of an elevation of angiotensin II levels are well known and include peripheral and coronary vasoconstriction, stimulation of growth factors [21], release of endothelin [22] and plasminogen activator inhibitor [23], and oxidation of low density lipoprotein [24]. The effect of increased aldosterone production has, however, received less attention and will be briefly reviewed. An escape of aldosterone despite the use of an ACE inhibitor has several potentially important consequences that could result in the relatively high incidence of morbidity and mortality seen in patients with symptomatic left ventricular dysfunction despite treatment with an ACE inhibitor. Aldosterone would be expected to play a role in recurrent heart failure through its well-known effects in promoting distal renal tubular sodium absorption [25]. Elevated levels of aldosterone have also been implicated, at least in animal models, in myocardial collagen production [26] and ventricular hypertrophy [27]. Increased myocardial collagen production and consequent left ventricular hypertrophy could lead to an impairment in diastolic filling, diastolic dysfunction, and decreased coronary flow reserve, and eventually systolic dysfunction and recurrent heart failure.

Aldosterone may also be of importance in the relatively high incidence of sudden cardiac death found in patients with both ischemic and nonischemic heart failure. Increased aldosterone levels cause a loss of potassium and magnesium from the distal renal tubule. Both hypokalemia and hypomagnesemia predispose to recurrent ventricular arrhythmias, digitalis toxicity, and therefore sudden cardiac death $[28,29]$. The use of adjunctive potassium is common in patients with heart failure treated with diuretics and digitalis; hypokalemia nevertheless remains a major problem. In part, the hypokalemia can be corrected by the concomitant use of magnesium, which facilitates potassium retention [30]. Aldosterone may also predispose to ventricular arrhythmias through its effect on norepinephrine uptake [31].

Barr et al. have recently shown that norepinephrine uptake, as assessed by the myocardial uptake of meta-iodobenzylguanidine ( $\mathrm{mIBG}$ ), is depressed in patients with heart failure and that this can be improved by the use of the aldosterone receptor antagonist spironolactone despite the use of an $\mathrm{ACE}$ inhibitor [31]. The finding in the SOLVD treatment trial [2] that sudden cardiac death was only minimally reduced by 
enalapril and the failure of type I antiarrhythmic agents to reduce mortality in patients with left ventricular dysfunction in the Cardiac Arrhythmia Suppression Trial (CAST) [32] are an impetus to develop further strategies to prevent ventricular arrhythmias and sudden cardiac death in patients with left ventricular dysfunction. Although there is considerable controversy regarding the effectiveness of ACE inhibitors in reducing sudden cardiac death in that sudden cardiac death was found to be reduced in the V-HeFT II trial [33], sudden cardiac death nevertheless remains an important cause of death in patients with symptomatic left ventricular dysfunction treated with an ACE inhibitor.

An escape of aldosterone could also play a role in recurrent ischemic events in patients with left ventricular dysfunction. The SOLVD trial pointed out the importance of recurrent ischemic events, myocardial infarction, and hospitalization for unstable angina in patients with left ventricular dysfunction due to ischemic as well as nonischemic cardiomyopathy [6]. Patients wtih recurrent ischemic events had a severalfold increase in the risk of death compared with patients without a recurrent ischemic event. Prior to this observation most clinicians focused on symptomatic improvement in patients with left ventricular dysfunction. The finding that recurrent ischemic events are common in both patients with ischemic as well as nonischemic cardiomyopathy has promoted efforts to prevent recurrent ischemia. Risk factor reduction in patients with ischemic cardiomyopathy, and correction of recurrent ischemia by revascularization, deserve increased emphasis.

Of importance in the SOLVD treatment [2-6] and prevention trials [34], as well as in the SAVE trial [4], was the finding that ACE inhibitors were effective in preventing recurrent myocardial infarction. These findings are of particular importance both for the patient with symptomatic left ventricular dysfunction as well as for the secondary prevention of ischemic heart disease in patients with asymptomatic left ventricular dysfunction, and have prompted a randomized trial to explore the role of ACE inhibitors in patients with ischemic heart disease and preserved left ventricular dysfunction $(\mathrm{EF} \geq 40 \%)$ [35]. While the effectiveness of ACE inhibitors in preventing recurrent ischemic events can be explained by their effect in preventing angiotensin II production, there is evidence to suggest that aldosterone production might also be of importance and that an escape of aldosterone could predispose to recurrent myocardial infarction.

As mentioned earlier, aldosterone has been shown to cause left ventricular hypertrophy in experimental animals [26] and is significantly correlated with left ventricular mass in patients [36]. Left ventricular hypertrophy has been shown to be associated with a decrease in coronary flow reserve [37]. A decrease in coronary flow reserve has been demonstrated in both patients with nonischemic and ischemic cardiomyopa- thy [38]. Prevention of left ventricular hypertrophy by ACE inhibitors and/or aldosterone receptor antagonists could be expected to maintain coronary flow reserve and thereby prevent recurrent myocardial ischemia.

There are, however, several other mechanisms whereby aldosterone could cause myocardial ischemia and infarction. Patients with primary aldosteronism have been found to have endothelial dysfunction, as evidenced by a decrease in forearm vascular response to the administration of acetylcholine as compared with normal individuals [39]. Removal of the aldosterone-producing tumor restores endothelial function. Endothelial dysfunction could be expected to lead to increased peripheral and coronary arterial resistance in response to neurohumoral stimuli such as angiotensin II, norepinephrine, or release of platelet products such as thromboxane and serotonin, all of which are endothelial-dependent vasodilators. Endothelial dysfunction has been suggested to be an important precursor of atherosclerosis [40] and has been demonstrated in patients with heart failure [41]. Release of endothelin from the endothelium has been shown to increase aldosterone levels [42]. Endothelin is a potent growth factor stimulant [43] and could play a role in the development of atherosclerosis [44] directly and/or through release of aldosterone.

Aldosterone has also been inversely correlated with serum high density lipoprotein cholesterol (HDL-C) concentrations [44]. A reduction in HDL-C concentration would be expected to predispose to atherosclerosis. A further potential link between aldosterone and atherosclerosis comes from the demonstration that there are specific aldosterone receptors on monocytes and that aldosterone administration results in an increase in intracellular monocyte calcium, sodium, and potassium $[46,47]$. The effect of these intracellular changes on electrolyte concentration are uncertain but could potentially influence monocyte function and/or cytokine release.

Aldosterone might also predispose to recurrent ischemia and infarction through its effects on magnesium. Individuals living in areas with low magnesium concentrations in their water have a significantly higher incidence of myocardial infarction than individuals living in areas with a high magnesium content [29]. Magnesium depletion in experimental animals has been shown to result in an increase in coronary vasomotor tone [47], and experimental hypomagnesemia has been used to cause coronary arterial spasm [48]. Experimental magnesium depletion has also been shown to cause cytokine release, which could further depress myocardial function $[49,50]$. Patients with symptomatic left ventricular dysfunction treated with high doses of loop diuretics activate their renin angiotensin-aldosterone system [51] and would be expected to have magnesium depletion. The incidence of magnesium depletion in patients with symptomatic left ventricular dysfunction treated with loop diuretics 
is, however, controversial. Recent studies have not detected a high incidence of hypomagnesemia in patients with chronic heart failure despite the use of loop diuretics [52,53]. While hypomagnesemia does not appear to be common, despite the use of loop diuretics, there is evidence for a decrease in intracellular magnesium concentrations, which may not be reflected in serum levels [54]. The administration of magnesium supplementation to patients with chronic heart failure treated with loop diuretics has been shown to result in a significant reduction in complex ventricular arrhythmias [55].

Due to the mechanisms outlined earlier and the finding in the CONSENSUS trial that there was a significant correlation between aldosterone and mortality [56], it has been postulated that an aldosterone receptor blocking agent would be of benefit in reducing the incidence of recurrent heart failure, ventricular arrhythmias, ischemic events, and therefore death in patients with heart failure despite the use of conventional therapy including an ACE inhibitor. There is evidence that the addition of spironolactone, an aldosterone receptor antagonist, to the therapy for patients with severe heart failure treated with conventional therapy including diuretics, digoxin, and an ACE inhibitor results in increased diuresis and symptomatic improvement [57]. A high incidence of hyperkalemia has not been observed in these patients despite concomitant treatment with an ACE inhibitor, nor in previous studies of patients with hypertension treated with an ACE inhibitor and aldactone. Nevertheless, the relative paucity of data on the safety of spironolactone in patients with symptomatic left ventricular dysfunction treated with an ACE inhibitor has prompted a prospective blinded randomized international multicenter study in order to explore its safety when used in conjunction with an ACE inhibitor, the Randomized Aldactone Evaluation Study (RALES Pilot Study). The data from this pilot safety study will be used to develop the dosing strategy for a subsequent study (RALES Mortality Trial) testing the efficacy of aldactone in reducing total mortality in patients with NYHA class IV heart failure treated with conventional therapy including an ACE inhibitor.

\section{Acknowledgments}

The author thanks Drs. John Alexander, Alfonso Perez, and Raphael Naudin of Searle Pharmaceuticals for their encouragement and help in developing the concepts and design of the RALES pilot trial.

\section{References}

1. The CONSENSUS Trial Study Group. Effects of enalapril on mortality in severe congestive heart failure. $N$ Engl $J$ Med 1987;316:1429-1435.
2. SOLVD Investigators. Effect of enalapril on survival in patients with reduced left ventricular ejection fraction and congestive heart failure. $N$ Engl $J$ Med 1991;295:293-302.

3. Cohn JN, Johnson G, Ziesche S, et al. A comparison of enalapril with hydralazine-isosorbide dinitrate in the treatment of chronic congestive heart failure. $N$ Engl $J \mathrm{Med}$ 1991;325:303-310.

4. Pfeffer MA, Braunwald E, Moye LA, et al. Effect of captopril on mortality and morbidity in patients with left ventricular dysfunction after myocardial infarction. $N$ Engl $J \mathrm{Med}$ 1992;327:669-677.

5. The Acute Infarction Ramipril Efficacy (AIRE) Study Investigators. Effect of ramipril on mortality and morbidity of survivors of acute myocardial infarction with clinical evidence of heart failure. Lancet 1993;342:821-828.

6. Yusuf S, Pepine CJ, Garces C, et al. Effect of enalapril on myocardial infarction and unstable angina in patients with low ejection fractions. Lancet 1992;340:1173-1178.

7. Captopril Multicenter Research Group. A placebo-controlled trial of captopril in refractory chronic congestive heart failure. J Am Coll Cardiol 1983;2:755-763.

8. The Captopril-Digoxin Multicenter Research Group. Comparative effects of therapy with captopril and digoxin in patients with mild-to-moderate heart failure. JAMA 1988; 259:539-544.

9. Garg R, Packer M, Pitt B, Yusuf S. Heart failure in the 1990s: Evolution of a major public health problem in cardiovascular medicine. $J$ Am Coll Cardiol 1993;22:3A-5A.

10. Staessen J, Lijnen P, Fagard R, et al. Rise in plasma concentration of aldosterone during long-term angiotensin II suppression. $J$ Endocrinol 1981;91:457-465.

11. Mareyev V, Skvortsov A, Masenko V, Belenkov Y. Escape of ACE inhibitor effects on aldosterone during long-term treatment of congestive heart failure. Abstract to be presented at the International Meeting on Heart Failure, Amsterdam, April 1995.

12. Aldigier JC, Huang $\mathrm{H}$, Dalmay $\mathbf{F}$, et al. Angiotensinconverting enzyme inhibition does not suppress plasma angiotensin II increase during exercise in humans. $J$ Cardiovasc Pharmacol 1993;21:289-295.

13. Borghi C, Boschi S, Ambrosioni E, Melandri G, Branzi A, Magnani B. Evidence of a partial escape of reninangiotensin-aldosterone blockade in patients with acute myocardial infarction treated with ACE inhibitors. J Clin Pharmacol 1993;33:40-45.

14. Pouleur H, Konstam MA, Benedict CR, et al. Progression of left ventricular dysfunction during enalapril therapy: Relationship with neuro-hormonal reactivation. Circulation 1993;88:I-293.

15. Husain A. The chymase-angiotensin system in humans. $J$ Hypertens 1993;11:1155-1159.

16. Dzau VJ. Vascular renin angiotensin pathways: A new therapeutic target. $J$ Cardiovasc Pharmacol 1992;10(Suppl 7): S13-S26.

17. Weber KT, Villarreal D, Griffing GT. Heart Failure: Pathophysiology, Aldosterone and Anti-aldosterone therapy. GD Searle \& Co., 1993.

18. Robertson JIS, Nicholls MG. The Renin-Angiotensin System. London: Gower Medical, 1993.

19. Davis JO, Hartroft PM, Titus EO, et al. The role of the renin-angiotensin system in the control of aldosterone secretion. J Clin Invest 1962;41:378-389.

20. Muller J. Spironolactone in the management of congestive cardiac failure: A review. Clin Ther 1986;9:63-76. 
21. Naftilan AJ, Pratt RE, Eldridge CS, et al. Angiotensin II induces c-fos expression in smooth muscle cell via transcriptional control. Hypertension 1989;13:706-711.

22. Dohi Y, Hahn AWA, Boulanger CM, et al. Endothelin stimulated by angiotensin II augments contractility of spontaneously hypertensive rat resistance arteries. Hypertension 1992;19:131-137.

23. Rydzewski B, Zelezna B, Tang W, et al. Angiotensin II stimulation of plasminogen activator inhibitor-I gene expression in astroglial cells from the brain. Endocrinology 1992;130:1255-1262.

24. Keidar S, Brook JG, Aviram M. Angiotensin II enhances lipid peroxidation of low-density lipoprotein. News Physiol Sci 1998;8:245-248.

25. Hall $\mathrm{JE}$, Brands MW. Intrarenal and circulating angiotensin II and renal function. In: Robertson JIS, Nicholls MG, eds. The Renin-Angiotensin System. Gower Medical, London, 1993.

26. Weber KT, Brilla CG. Pathological hypertrophy and cardiac interstitium: Fibrosis and renin-angiotensin-aldosterone system. Circulation 1991;83:1849-1865.

27. Weber KT, Villareal D. Aldosterone and antialdosterone therapy in congestive heart failure. Am J Cardiol 1993;71: $3 \mathrm{~A}-11 \mathrm{~A}$.

28. Wester PO, Dyckner T. Intracellular electrolytes in cardiac failure. Acta Med Scand 1986;707:33-36.

29. Eisenberg MJ. Magnesium deficiency and sudden death. Am Heart $J$ 1992;124:544-549.

30. Whang R, Flink EB, Dyckner T, Wester PO, Aikawa JK, Ryan MP. Magnesium depletion as a cause of refractory potassium repletion. Arch Intern Med 1985;145:1686-1689.

31. Barr CS, Hanson J, Kennedy N, Lang CC, Struthers AD. The effect of a mineralocorticoid antagonist on myocardial mIBG uptake in congestive heart failure. Circulation 1993; 88:I-256.

32. Cardiac Arrhythmia Suppression Trial (CAST) Investigators. Preliminary report: Effect of encainide and flecainide on mortality in randomized trial of arrhythmia suppression after myocardial infaretion. N Engl J Med 1989;321:406-412.

33. Goldman S, Johnson G, Cohn JN, Cintron G, Smith R, Francis G, for the V-HeFT VA Cooperative Studies Group. Mechanism of death in heart failure. The vasodilator-heart failure trials. Circulation 1993;87:VI24-VI-31.

34. The SOLVD Investigators. Effect of enalapril on mortality and the development of heart failure in asymptomatic patients with reduced left ventricular ejection fraction. $N$ Engl $J$ Med 1992;327:685-691.

35. Texter M, Lees RS, Pitt B, et al. The Quinapril Ischemic Event Trial (QUIET) design and methods: Evaluation of chronic ACE inhibitor therapy after coronary artery intervention. Cardiovasc Drugs Ther 1993;7:273-282.

36. Bauwens FR, Duprez DA, DeBuyzere ML, et al. Influence of the arterial blood pressure and nonhemodynamic factors on left ventricular hypertrophy in moderate essential hypertension. Am J Cardiol 1991;68:925-929.

37. Karam R, Healy BP, Wicker P. Coronary reserve is depressed in postmyocardial infarction reactive cardiac hypertrophy. Circulation 1990;81:238-246.

38. Yuen JL, Wu J, Nanna M, et al. Coronary vascular reserve is substantially reduced in dilated cardiomyopathy independently from the presence or absence of coronary artery disease. J Am Coll Cardiol 1993;21:364A.

39. Taddei S, Virdis A, Mattei P, Salvetti A. Vasodilation to acetylcholine in primary and secondary forms of human hypertension. Hypertension 1993;21:929-933.
40. Harrison DG, Freiman PC, Mitchell GG, et al. Alterations of vascular reactivity in atherosclerosis. Circ Res 1987; 61(Suppl II):74-80.

41. Treasure CB, Vita JV, Cox DA, et al. Endotheliumdependent dilation of the coronary microvasculature is impaired in dilated cardiomyopathy. Circulation 1990;3: $772-779$.

42. Goetz KL, Wang BC, Madwed JF, Zhu IL, Leadley RJ. Cardiovascular, renal, and endocrine responses to intravenous endothelin in conscious dogs. Am J Physiol 1988;255: R1064-R1068.

43. Hirata Y, Takagi, Fukuda Y, Marumo F. Endothelin is a potent mitogen for rat vascular smooth muscle cells. Atherosclerosis 1989;78:225-228.

44. Lind L, Lithell H, Wide L, et al. Metabolic cardiovascular risk factors and the renin-aldosterone system in essential hypertension. J Hum Hypertens 1992;6:27-29.

45. Wehling $M$, Christ $M$, Theisen $K$. Membrane receptors for aldosterone: A novel pathway for mineralo-corticoid action. Endocrinol Metab 1992;26:E974-E979.

46. Wehling M, Armanini D, Strasser T, et al. Effect of aldosterone on sodium and potassium concentrations in human mononuclear leukocytes. Endocrinol Metab 1987;252: E505-E508.

47. Turlapaty PDMV, Altuna BM. Magnesium deficiency produces spasms of coronary arteries: Relationship to etiology of sudden death ischemic heart disease. Science 1989;208: 198-200.

48. Miyagi H, Yasue H, Okumura K, et al. Effect of magnesium on anginal attack induced by hyperventilation in patients with variant angina. Circulation 1989;79:597-602.

49. Weglicki WB, Phillips TM, Freedman AM, Cassidy MM, Dickens BF. Magnesium-deficiency elevates circulating levels of inflammatory cytokines and endothelin. Mol Cell Biochem 1992;110:169-173.

50. Finkel MS, Oddis CV, Jacob TD, Watkins SC, Hattler BG, Simmons RL. Negative inotropic effects of cytokines on the heart mediated by nitric oxide. Science 1992;257:387-389.

51. Francis GS, Benedict C, Johnstone DE, et al. Comparison of neuroendocrine activation in patients with left ventricular dysfunction with and without congestive heart failure. Circulation 1990;82:1724-1729.

52. Gottlieb SS. Importance of magnesium in congestive heart failure. Am J Cardiol 1989;63:39G-42G.

53. Ralston MA, Murnane MR, Kelley RE, Altschuld RA, Unverferth DV, Leier CV. Magnesium content of serum, circulating mononuclear cells, skeletal muscle, and myocardium in congestive heart failure. Circulation 1989;80:573-580.

54. Dorup I, Skajaa K, Clausen T, Kjeldsen K. Reduced concentrations of potassium, magnesium and sodium-potassium pumps in human skeletal muscle during treatment with diuretic. Br Med $J$ 1988;296:455-458.

55. Bashir Y, Sneddon JF, Staunton HA, et al. Effects of longterm oral magnesium chloride replacement in congestive heart failure secondary to coronary artery disease. $A m J$ Cardiol 1993;72:1156-1162.

56. Swedberg K, Eneroth P, Kjekshus J, Wilhelmsen L, for the CONSENSUS Trial Study Group. Hormones regulating cardiovascular function in patients with severe congestive heart failure and their relation to mortality. Circulation 1990;82:1730-1736.

57. van Vliet AA, Donker AJM, Nauta JJP, et al. Spironolactone in congestive heart failure refractory to high-dose loop diuretic and low-dose angiotensin converting enzyme inhibitor. Am J Cardiol 1993;71:21A-28A. 